\title{
Perimenopausal 6 to 12 Months Since LMP
}

National Cancer Institute

\section{Source}

National Cancer Institute. Perimenopausal 6 to 12 Months Since LMP. NCI Thesaurus.

Code C158829.

A time period of 6-12 months since the last menstrual period. 